\title{
Reinforcement Calculation of Circular and Annular Cross Section
}

\author{
Yao KAICHENG, Zhou DONGHUA*, Shuang CHAO, Lan SHUWEI, Chen JIN
}

\begin{abstract}
Circular and annular cross-section is one of the more common cross-section forms, such as water towers, piers, etc. But reinforcement calculation of these crosssections is dual, the nonlinearity in width change and the nonlinear stress-strain relationship of concrete and reinforcement, which brings inconvenience to the calculation. In this paper, the corresponding calculation formulas are analytically derived and practical design charts (nomograms) are obtained. Based on stress-stain curve of concrete and reinforcement, five strain distribution regions were constructed. Via strains stress can be determined and then via the stress internal force can be computed, without application of the traditional equivalent rectangular stress block in concrete compression zone. The obtained dimensionless design charts can be applied to all load cases, such as uniform tension, tension with a small and large eccentricity, pure bending, compression with a large and small eccentricity and uniform compression. The calculation by using the design charts is simple and quick.
\end{abstract}

Keywords: axial and eccentric load; circular and annular cross-section; dimensionless design charts; reinforcement ring; strain region

\section{INTRODUCTION}

Members with circular or annular section shapes have low resistance to fluid (wind, water) and are commonly used in engineering structures, such as piers, water towers, etc. Analytical calculating reinforcement area of the circular and annular cross sections is generally needed to solve transcendental equations, which are provided in some specifications, for example in chinses code [1-2]. Nevertheless the application of these equations shows some shortcomings: (1) solutions can only be iteratively solved with software and cannot be computed by hand, and this is inconvenient for practice; (2) The transcendental equations are derived often with strong simplifications (e.g. in reference 1), which make the scope of application limited and it is applicable only when the neutral axis is within the cross section. For example, in the reference [3, 4, 14-19], the authors derived the formulas, which are all based on the equivalent rectangular stress block. Among them some are obtained analytical formulas [16-19] and some others are numerical calculations [14, 15]. To overcome these shortcomings some design chats are made in this paper for quick calculating reinforcement area or for checking strength of the circular and annular cross sections. For more accuracy instead of the rectangular stress block, the stress-strain curves of concrete and reinforcement adopted in reference lare applied to derive formulas. Although nowadays computers are widely used, quick calculation tools to determine reinforcement area of circular and annular cross sections are still needed and welcome, which enable a hand calculation not only for quick determining reinforcement area, but also for quick checking the results computed by computer.

\section{THE METHOD, DERIVATION AND DESIGN CHARTS \\ 2.1 Stress-Strain Relationship of Concrete and Reinforcement}

The following stress-strain curves of concrete and reinforcement are used and shown in Fig. 1.

The mathematical expression of the constitutive curves in Fig. 1 is given as the following:

$$
\begin{aligned}
& \left\{\begin{array}{lr}
\sigma_{c}=\left(-\varepsilon_{c}-0.25 \varepsilon_{c}^{2}\right) f_{c} & \left(0>\varepsilon_{c}>-2.0 \%\right) \\
\sigma_{c}=f_{c} & \left(-2.0 \% \mathrm{o} \geq \varepsilon_{c} \geq-3.3 \%\right)
\end{array}\right. \\
& \begin{cases}\sigma_{s}=E_{s} \varepsilon_{s} & \left(0<\varepsilon_{s}<\varepsilon_{y}\right) \\
\sigma_{s}=\sigma_{y} & \left(\varepsilon_{y} \leq \varepsilon_{s} \leq 10 \% \mathrm{o}\right)\end{cases}
\end{aligned}
$$

where $\varepsilon_{c}$ is strain of concrete and $\varepsilon_{s}$ strain of reinforcement. The ultimate strain limit of concrete and reinforcement is $-3,3 \%$ and $10 \%$ respectively.

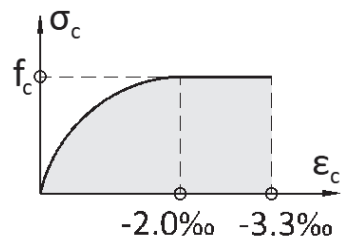

(a)

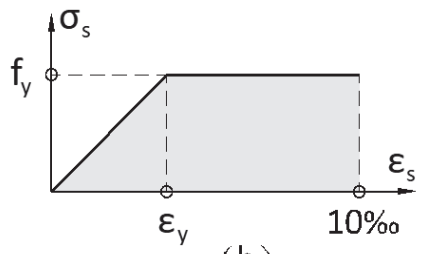

(b)
Figure 1 Constitutive relation (a) concrete,(b) reinforcement

It should be noted that all strain values take the values before the symbol of per thousand, i.e. are not divided by 1000 .

\subsection{Division of the Whole Possible Strain Distributions Into 5 Strain Regions}

The main process of the method in the paper is variation of possible strains distributions of cross section, and then via the strains corresponding stresses can be valued according to constitutive curves of concrete and reinforcement and finally the internal forces are computed. Therefore, it is necessary to know how and in which region the strain distributions can be changed. Based on the stressstrain curves in Fig. 1 the strain distributions of cross section can be divided into five strain regions as shown in Fig. 2. Within each strain region there are always strains on one side (top section edge or bottom reinforcement) fixed in the ultimate strain limit state, and on the other side the strains can be varied, thus it can be ensured that all strain distributions are in the ultimate limit state, and this means: 
the section can be assumed either tensile failure, or compression failure, or balanced failure occurred [4-13].

After dividing the whole possible strain distributions into 5 regions, the strains become now known, and then the corresponding stresses can be determined via the constitutive relationships of concrete and reinforcement. By integration of the stresses over the cross section the internal normal forces and bending moments can be obtained.

The characteristics of each strain region are briefly described as follows:

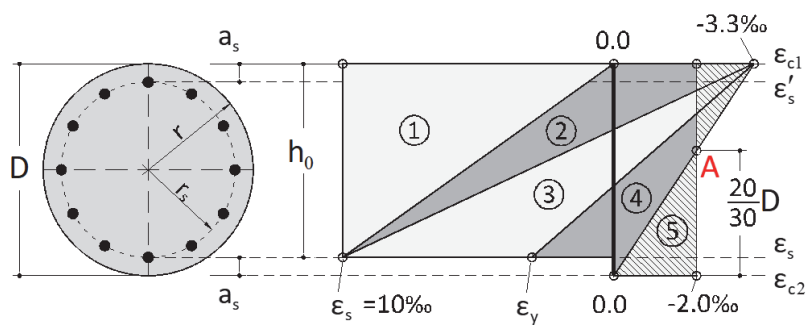

Figure 2 Strain change region of concrete and reinforcement

Region (1): Holding the bottom strain of reinforcement at the value of $\varepsilon_{s}=10 \%$, the top edge strain of the cross section can be varied ( $\varepsilon_{c 1}=10 \sim 0 \%$ ). Since the full cross section being in tension, the tensile strength of concrete is neglected, and the tension force in cross section is carried out only by reinforcement. At the left boundary of the region the strain distribution represents uniform tension, at which the values of the steel strain on both sides reach the value of $\varepsilon_{s}=10 \%$ and the corresponding curvature is 0 . The load cases of the region may be uniform tension, tension with a small eccentricity, and the curvature increases gradually from zero.

Region (2): Keeping the bottom strain of reinforcement fixed at the value $\varepsilon_{s}=10 \%$, and the top edge strain of concrete continuing varied with the value $\varepsilon_{c 1}=0 \sim-3,3 \%$, the neutral axis moves into the cross section and the curvature increases further. When the top edge strain reaches $\varepsilon_{c 1}=-3,3 \%$, the curvature becomes the maximum. The load cases of the region may be tension or compression with eccentricity.

Region (3): Fixing the top edge strain at the value of $\varepsilon_{c 1}=-3,3 \%$, and the bottom strain of reinforcement can be varied with the value $\varepsilon_{s}=10 \% \sim \varepsilon_{y}$. As the bottom strain gradually decreases, the neutral axis moves down, and the depth of the compression zone increases and the curvature decreases. The load cases of the region may be pure bending and compression with a large eccentricity.

Region (4): the top edge concrete strain keeping on fixed at the value $\varepsilon_{c 1}=-3,3 \%$, the bottom reinforcement strain continues to decrease and even enters the compression zone, until the bottom edge strain of concrete reduces to $\varepsilon_{c 2}=0$. The neutral axis reaches the bottom edge of the cross section and the curvature is reduced further. The load cases of the region may be compression with a small eccentricity.

Region (5): This region is a swept region where the right boundary line of the region (4) is rotated counterclockwise around point A to the vertical position (point A can be calculated from the geometric relationship with respect to the top and bottom edge strains). The top edge strain of the cross section reduces from $-3,3 \%$ to $-2,0 \%$ and the bottom edge strain increases from $0,0 \%$ to $-2,0 \%$ simultaneously, and the curvature decreases to zero The load cases of the region may be compression with a small eccentricity and uniform compression.

So far, the five divided strain regions contain all possible strain distributions and cover all load cases known as uniform tension, tension with a small and large eccentricity, pure bending, compression with a large and small eccentricity and uniform compression. It is incidental to note that in regions (2) and (5) the top edge strains of the cross section are variable and hence the equivalent rectangular stress block cannot be applied.

\subsection{Computation Cross Section Stress and Internal Force}

Using the geometric relationship of the strain distribution in Fig. 3 the depth of the compression zone $x$ can be calculated:

$$
x=\frac{\varepsilon_{c 1}}{\varepsilon_{c 1}-\varepsilon_{c 2}} d
$$

When $x$ is obtained, it is able to determine the strain $\varepsilon_{c i}$ at any fiber within the cross section.

$\varepsilon_{c i}=\frac{z}{x} \varepsilon_{c 1}$

where $z$ can be obtained from neutral depth $x$ subtracting chord height of the circle $h_{i}$, see Eq. (5)

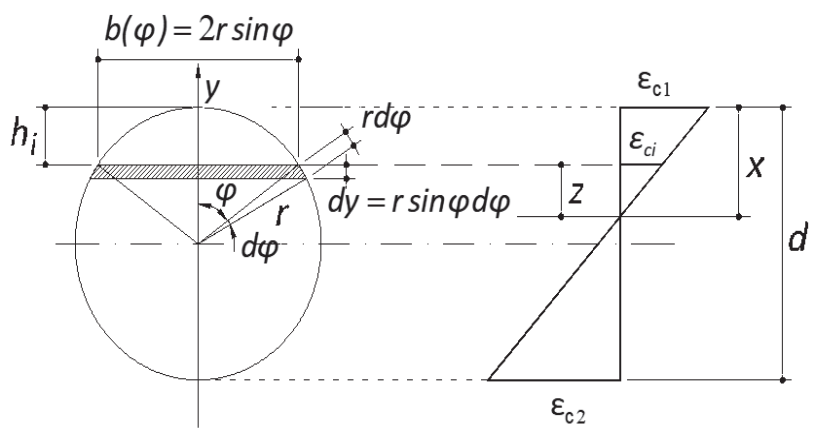

Figure 3 Section parameters and strain

The chord height $h_{i}=r(1-\cos \varphi)$ reaches the minimum of zero at the top of the circle, and the maximum of $2 r$ at the bottom of the circle.

$$
z=x-h_{i}=x-r(1-\cos \varphi)
$$

In Eq. (5) variables $x$ and $h_{i}$ are independent, $x$ varies with the cross section edge strain, but $h_{i}$ varies with central angle $\varphi$, which increases from top to bottom of the circle. If $x$ is fixed, $z$ varies only with the chord height, and hence varies also with the central angle $\varphi$, thus the strain of any 
fiber can be described as a function of the central angle. Substituting value $z$ of Eq. (5) into Eq. (4).

$$
\varepsilon_{c i}=\frac{x-r(1-\cos \varphi)}{x} \varepsilon_{c 1}
$$

and then substituting value $\varepsilon_{c i}$ of Eq. (6) into Eq. (1), the stress of any fiber can be calculated.

$$
\begin{aligned}
& \sigma_{c i}(\varphi)=-\left(\varepsilon_{c i}+0.25 \varepsilon_{c i}^{2}\right) f_{c}= \\
& =\left\{\begin{array}{l}
-\left(\frac{r}{x}-1\right)^{2} \frac{\varepsilon_{c 1}^{2}}{4}-\left(\frac{r}{x}-1\right) \varepsilon_{c 1}+ \\
+\left[\frac{r}{x}\left(\frac{r}{x}-1\right) \frac{\varepsilon_{c 1}^{2}}{2}+\frac{r}{x} \varepsilon_{c 1}\right] \cos \varphi-\frac{r^{2}}{x^{2}} \frac{\varepsilon_{c 1}^{2}}{K_{3}} \cos ^{2} \varphi
\end{array}\right\} f_{c}= \\
& =\left(K_{1}+K_{2} \cos \varphi+K_{3} \cos ^{2} \varphi\right) f_{c}
\end{aligned}
$$

After getting the expression of stress, the stress can be integrated over the cross section to obtain the internal axial force and bending moment. The calculated bending moment is about the horizontal centroid axis of the circle. For easy calculation, the stress block in Fig. 4 should be divided into two parts, one part consisting of rectangle and another of parabola.

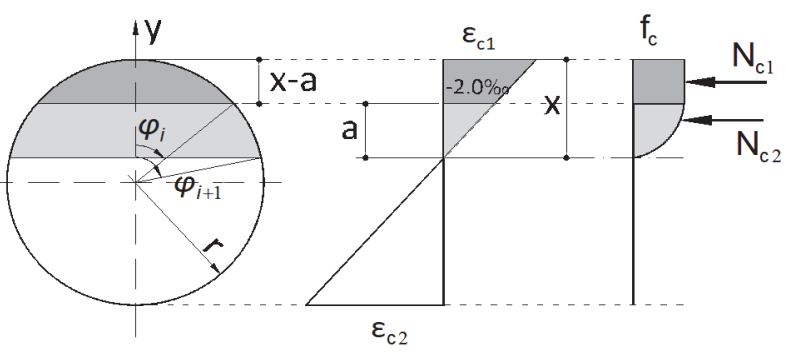

Figure 4 Strain and stress distribution (rectangle and parabola)

\subsubsection{Rectangular Stress Block $\left(\varepsilon_{c 1}=-2.0 \% \sim-3.3 \%\right.$ )}

The concrete stress is constant, and the axial force and bending moment can be computed by integration of stress over the cross section, as shown in Eq. (8) and Eq. (9).

$$
\begin{aligned}
N_{c 1} & =\int_{0}^{x}-f_{c} b(\varphi) \mathrm{d} y=-2 r^{2} f_{c} \int_{0}^{x} \sin ^{2} \varphi \mathrm{d} \varphi= \\
& =-2 f_{c} r^{2}\left[\frac{\varphi}{2}-\frac{\sin 2 \varphi}{4}\right]_{0}^{\varphi_{j}}
\end{aligned}
$$

$M_{c 1}=\int_{0}^{x}-f_{c} b(\varphi) \cdot r \cos \varphi \mathrm{d} y=$

$=-2 r^{3} f_{c} \int_{0}^{x} \sin ^{2} \varphi \cos \varphi \mathrm{d} \varphi=-\frac{2}{3} f_{c} r^{3}\left[\sin ^{3} \varphi\right]_{0}^{\varphi_{j}}$

\subsubsection{Parabolic Stress Block $\left(\varepsilon_{c 1}=0 \% \sim-2.0 \%\right)$}

Since the strains in this part are variable and the stresses are accordingly not constant, their values can be determined by Eq. (7). After integration of stress the corresponding axial force and bending moment can be obtained.

$$
\begin{aligned}
N_{c 2} & =\int_{0}^{x} \sigma_{c i}(\varphi) b(\varphi) \mathrm{d} y=2 r^{2} \int_{0}^{x} \sigma_{c i}(\varphi) \sin ^{2} \varphi \mathrm{d} \varphi= \\
& =2 r^{2} \int_{0}^{x}\left(K_{1}+K_{2} \cos \varphi+K_{3} \cos ^{2} \varphi\right) \sin ^{2} \varphi \mathrm{d} \varphi= \\
& =2 f_{c} r^{2}\left[\begin{array}{l}
\left(\frac{\varphi}{2}-\frac{\sin 2 \varphi}{4}\right) K_{1}+\frac{\sin ^{3} \varphi}{3} K_{2} \\
\left.+\left(-\frac{\sin \varphi \cos ^{3} \varphi}{4}+\frac{\varphi}{8}-\frac{\sin 2 \varphi}{16}\right)\right]_{\varphi_{j}}^{\varphi_{j+1}}
\end{array}\right.
\end{aligned}
$$

$M_{c 2}=\int_{0}^{x} \sigma_{c i}(\varphi) b(\varphi) r \cos \varphi \mathrm{d} y=$

$=2 r^{3} \int_{0}^{x} \sigma_{c i}(\varphi) \sin ^{2} \varphi \cos \varphi \mathrm{d} \varphi=$

$=2 r^{3} \int_{0}^{x}\left(K_{1}+K_{2} \cos \varphi+K_{3} \cos ^{2} \varphi\right) \sin ^{2} \varphi \cos \varphi \mathrm{d} \varphi=$

$=2 f_{c} r^{3}\left[\begin{array}{l}\frac{\sin ^{3} \varphi}{3} K_{1}+\left(-\frac{\sin \varphi \cos ^{3} \varphi}{4}+\frac{\varphi}{8}-\frac{\sin 2 \varphi}{16}\right) K_{2} \\ +\left(-\frac{\sin \varphi \cos ^{4} \varphi}{5}+\frac{\sin \varphi}{5}-\frac{\sin ^{3} \varphi}{15}\right) K_{3}\end{array}\right]_{\varphi_{j}}^{\varphi_{j+1}}$

Obviously, the axial force and bending moment obtained above are dependent on concrete strength and size of cross section. In order to get a more general dimensionless expression, Eq. (8) and Eq. (10) should be divided by $\pi r^{2} f_{c}$ and Eq. (9) and Eq. (11) by $\pi r^{3} f_{c}$ respectively, and then the dimensionless axial force and bending moment are obtained as follows:

$$
\begin{aligned}
& \left\{\begin{array}{l}
n_{c 1}=-\frac{2}{\pi}[\varphi-0.5 \sin 2 \varphi]_{0}^{\varphi_{j}} \\
m_{c 1}=-\frac{2}{3 \pi}\left[\sin ^{3} \varphi\right]_{0}^{\varphi_{j}} \\
n_{c 2}=\frac{2}{\pi}\left[\begin{array}{l}
\left.\left(\frac{\varphi}{2}-\frac{\sin 2 \varphi}{4}\right) K_{1}+\frac{\sin ^{3} \varphi}{3} K_{2}+\right]^{\varphi_{j+1}} \\
\left.+\left(-\frac{\sin \varphi \cos ^{3} \varphi}{4}+\frac{\varphi}{8}-\frac{\sin 2 \varphi}{16}\right)\right]_{\varphi_{j}}
\end{array}\right.
\end{array}\right. \\
& m_{c 2}=\frac{2}{\pi}\left[\begin{array}{l}
\frac{\sin ^{3} \varphi}{3} K_{1}+\left(-\frac{\sin \varphi \cos ^{3} \varphi}{4}+\frac{\varphi}{8}-\frac{\sin 2 \varphi}{16}\right) K_{2}+ \\
+\left(-\frac{\sin \varphi \cos ^{4} \varphi}{5}+\frac{\sin \varphi}{5}-\frac{\sin ^{3} \varphi}{15}\right) K_{3}
\end{array}\right]_{\varphi_{j}}^{\varphi_{j+1}}
\end{aligned}
$$

It is to note that the values of integral interval of Eq. (12) have not been determined. Their determination depends on the shape of the stress blocks, which can be varied and result in the following 4 stress block shapes:
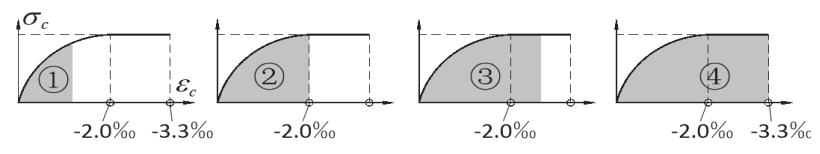

Figure 5 Shape cases of the concrete stress block

(1) a partial parabola; (2) a full parabola; (3) a full parabola + a partial rectangular block; (4) a full parabola + full rectangular block.

Taking case (1) as an example, the stress distribution is a partial parabola (see Fig. 5 and Fig. 6), and the strain 
increases gradually from zero $\left(0>\varepsilon_{c 1}>-2,0 \%\right.$ ). The integral interval begins from the top point of the circle to the neutral axis, which correspond a central angle $\varphi$, and its value can be calculated by Eq. (13).

$\left\{\begin{array}{l}\varphi_{i}=\arccos (q) \\ q=\left(r-h_{i}\right) / r\end{array}\right.$

The magnitude of the central angle is dependent on the corresponding chord height $h_{i}$, which varies with the stress block shape and size. For example, an angle can be calculated by substituting a chord height shown in Fig. 6 into Eq. (13). At the top point of the circle the chord height is zero, and at the neutral axis is $x$.

Taking Case (3) as another example, the stress block shape consists of a full parabola + a partial rectangular block (see Fig. 5 and Fig. 4), and its strain range is $-2,0 \%>\varepsilon_{c 1}>-3,3 \%$. The whole integral interval is to be divided into two intervals (a rectangular part and a parabolic part). The 1st interval starts from top point of the circle and ends at the junction point between rectangular and parabolic stress block. The $2^{\text {nd }}$ interval starts from junction point mentioned above and ends at the neutral axis. The chord heights of three boundary points are $0, x-a$ and $x$, and their $q$ - values are $1, r-(x-a) / r$ and $(r-x) / r$ respectively.

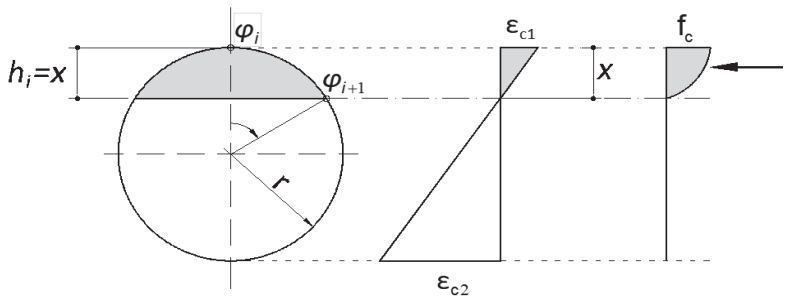

Figure 6 The upper and lower limits of the integral corresponding to the central angle (partial parabola)

Different strain regions result in different stress block shapes, and they can be summarized into three stress block shapes and their $q$ - values can be calculated according to Tab. 1.

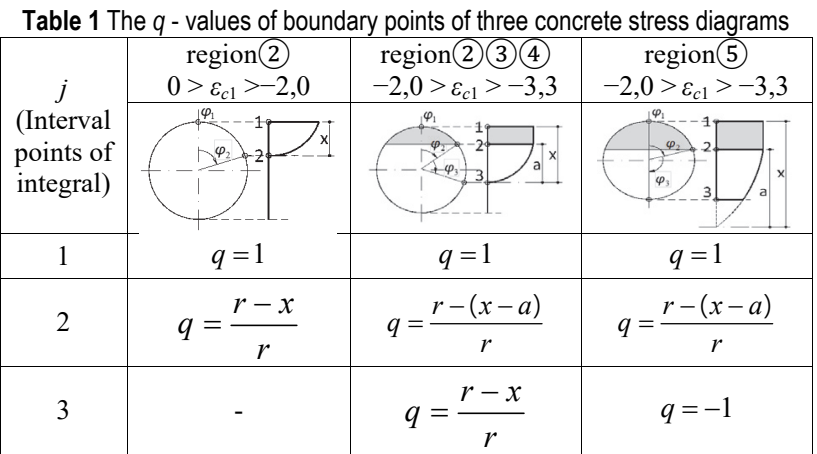

\subsection{Calculation of Stress and Internal Force of Reinforcement Ring}

The reinforcement is usually discretely distributed, which makes it inconvenient for calculation. For easy calculation, the reinforcement can be assumed to be continuously distributed, that is, the total area of the reinforcement is distributed on the circumference of reinforcement ring having a radius $r_{s}$.

$\bar{a}_{s}=\frac{A_{s}}{2 \pi r_{s}}$

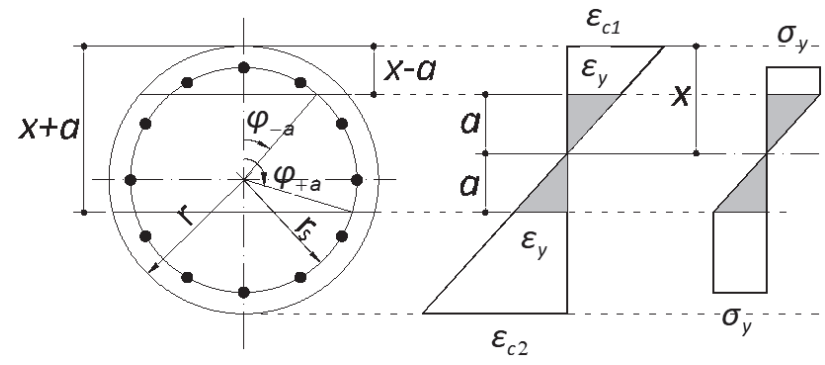

Figure 7 Strain and stress of reinforcement ring

Hereinafter, the continuously distributed reinforcement is referred to as a reinforcement ring. The stress block of the reinforcement ring (Fig. 7) can be also divided into two parts, one is a rectangular stress block (plastic zone) and another is a triangular stress block (elastic zone). The internal forces of the two stress blocks are calculated separately below.

\subsubsection{Rectangular Stress Block (Plastic Zone)}

In this part, the stress of the reinforcement ring is constant, and the calculation of the axial force and the bending moment is actually the calculation of area and area moment, as shown in Eq. (15) and Eq. (16).

$$
\begin{aligned}
& N_{s 1}=2 \int f_{y} \bar{a}_{s} r_{s} \mathrm{~d} \varphi=2 f_{y} \bar{a}_{s} r_{s c}[\varphi]_{0}^{\varphi_{j}} \\
& M_{s 1}=2 \int f_{y} \bar{a}_{s} r_{s} \cos \varphi r_{s} \mathrm{~d} \varphi= \\
& =2 f_{y} \bar{a}_{s} r_{s}^{2} \int \cos \varphi \mathrm{d} \varphi=2 f_{y} \bar{a}_{s} r_{s}^{2}[\sin \varphi]_{0}^{\varphi_{j}}
\end{aligned}
$$

\subsubsection{Triangular Stress Block (Elastic Zone)}

The strain and stress of the reinforcement ring at any fiber of cross section are:

$$
\begin{aligned}
& \varepsilon_{s i}=\frac{x-\left(r-r_{s} \cos \varphi\right)}{x} \varepsilon_{c 1} \\
& \sigma_{s i}=E_{s} \varepsilon_{s i}=E_{s} \frac{x-\left(r-r_{s} \cos \varphi\right)}{x} \varepsilon_{c 1}
\end{aligned}
$$

The resulting axial force in elastic zone can be obtained by integrating stress.

$$
\begin{aligned}
N_{s 2} & =2 \int \sigma_{s i} \bar{a}_{s} r_{s} \mathrm{~d} \varphi=2 E_{s} \bar{a}_{s} r_{s} \int \frac{x-\left(r-r_{s} \cos \varphi\right)}{x} \varepsilon_{c 1} \mathrm{~d} \varphi= \\
& =2 E_{s} \bar{a}_{s} \frac{r_{s}}{x} \varepsilon_{c 1}\left[(x-r) \varphi+r_{s} \sin \varphi\right]_{\varphi_{j}}^{\varphi_{j+1}}
\end{aligned}
$$


The resulting bending moment in elastic zone can be obtained by integrating stress multiplying lever arm about the horizontal center axis of the circle.

$$
\begin{aligned}
& M_{s 2}=2 \int \sigma_{s i} \bar{a}_{s} r_{s}^{2} \cos \varphi \mathrm{d} \varphi \\
& =2 E_{s} \bar{a}_{s} r_{s}^{2} \int \frac{x-\left(r-r_{s} \cos \varphi\right)}{x} \varepsilon_{c 1} \cos \varphi \mathrm{d} \varphi \\
& =2 E_{s} \bar{a}_{s} \frac{r_{s}^{2}}{x} \varepsilon_{c 1}\left[(x-r) \sin \varphi+r_{s}\left(\frac{\varphi}{2}+\frac{\sin 2 \varphi}{4}\right)\right]_{\varphi_{j}}^{\varphi_{j+1}}
\end{aligned}
$$

For easy using, it is better to employ the dimensionless internal force and bending moment. Therefore Eq. (15) and Eq. (19) are divided by $\pi r^{2} f_{c}$, and Eq. (16) and Eq. (20) by $\pi r^{3} f_{c}$,

$$
\left\{\begin{array}{l}
n_{s 1}=\frac{\omega_{s}}{\pi}[\varphi]_{0}^{\varphi_{j}} \\
m_{s 1}=\frac{\omega_{s}}{\pi} \frac{r_{s}}{r}[\sin \varphi]_{0}^{\varphi_{j}} \\
n_{s 2}=\frac{\omega_{s}}{\pi} \frac{E_{s}}{f_{y}} \frac{\varepsilon_{c 1}}{x}\left[(x-r) \varphi+r_{s} \sin \varphi\right]_{\varphi_{j}}^{\varphi_{j+1}} \\
m_{s 2}=\frac{\omega_{s}}{\pi} \frac{E_{s}}{f_{y}} \frac{\varepsilon_{c 1}}{x} \frac{r_{s}}{r}\left[(x-r) \sin \varphi+r_{s}\left(\frac{\varphi}{2}+\frac{\sin 2 \varphi}{4}\right)\right]_{\varphi_{j}}^{\varphi_{j+1}}
\end{array}\right.
$$

where $\omega_{s}$ is mechanical reinforcement ratio defined by:

$$
\omega_{s}=\frac{A_{s}}{\pi r^{2}} \frac{f_{y}}{f_{c}}
$$

The determination of the integral points in Eq. (21) is similar to that of concrete described before. Depending on the stress block shape of the reinforcement ring there are also four cases shown in Fig. 8:
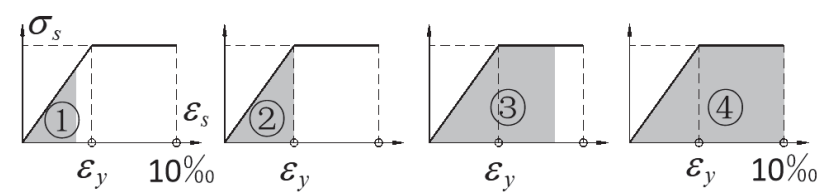

Figure 8 Shape cases of stress block of the reinforcement ring

Table 2 The $q$-values of boundary points of three stress diagrams of

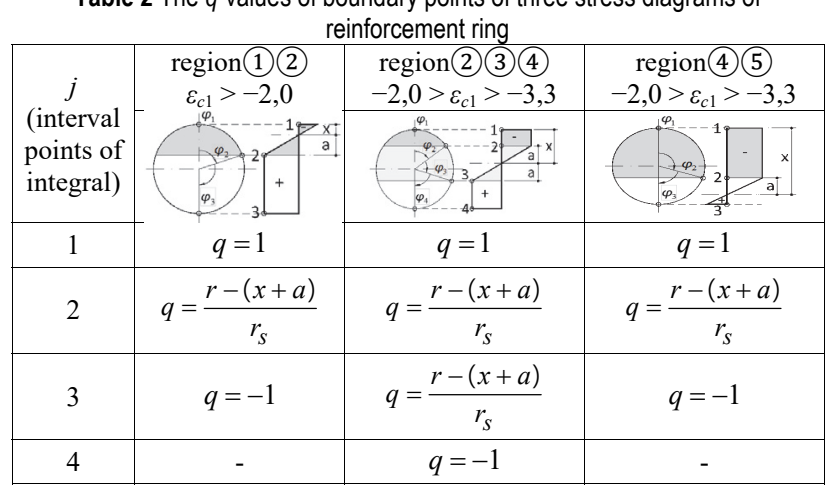

a partial triangle; (2) a full triangle; (3) a full triangle + a partial rectangle; (4) a full triangle + a full rectangle; Different strain regions have different stress shapes; they can be summarized into three cases. The calculation of $q$ values in each case can be carried out as shown in Tab. 2 .

\subsection{Calculation of Reinforcement of Annular Section}

The calculation of the annular cross section is basically the same as that of the circular cross section. The only difference is that it should be treated a little by calculating the internal force of the concrete, i.e. the compression area of the small circle (inner circle) must be subtracted from the area of the large circle (outer circle) (see Fig. 9).

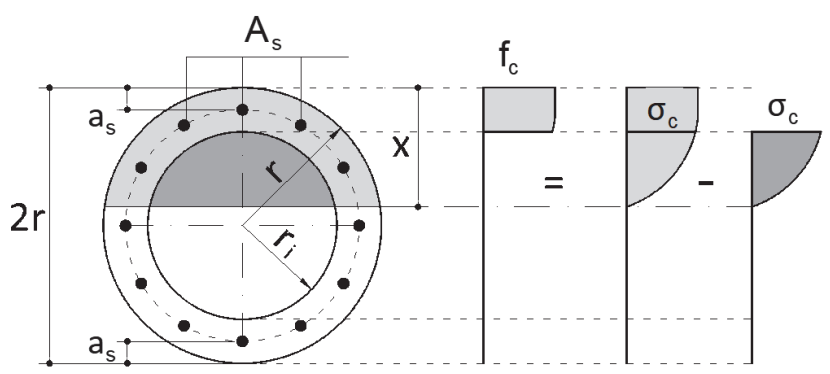

Figure 9 Compression zone of annular section

Hereto all formulae have been derived. The main idea of the method is to determine stress according to possible strain distributions in ultimate limit state, which are divided into 5 regions based on stress-strain relationship of concrete and reinforcement from Chinese Code. And then integration of stress results in axial force and bending moments.

Based on the five strain regions (see Fig. 2), the strains of concrete and reinforcement, as well as the areas of the reinforcement are varied. The corresponding dimensionless internal forces and bending moments are calculated. For practice usage, i.e. a quick calculating the reinforcement area of circular and annular cross section, all calculated values by using derived formulae are drawn into the design charts (nomograms) in Fig. 10. It is no need to solve iteratively transcendental equations given in the Chinese Code to get reinforcement area of circular and annual cross section. In addition some useful information can be obtained from Fig. 10:

(1) The balanced failure point of the circular cross section is no longer at the point of the maximum bending moment, while the balanced failure point of rectangular cross section indicates the maximum bending moment.

(2) The innermost curve of circular cross section $\left(\omega_{\mathrm{s}}=0.0\right)$ represents $m-n$ interaction curve of pure concrete having no reinforcement. Since the tensile strength of concrete is neglected, the curve is located above the horizontal axis and i.e. in the compression-bending zone. The other remaining $m-n$ curves having reinforcement begin to expand from the innermost curve. Therefore the graphic area above the horizontal axis is larger than that below the horizontal axis, in other words, the compression-bending zone is larger than the tension-bending zone. If the tensile stress of concrete was taken into account, the whole $m-$ 
ninteraction curves would be symmetrical to the horizontal axis.

(3) Comparing Fig. 10a with Fig. 10b, it is easy to see the difference that the curve of the innermost $\left(\omega_{\mathrm{s}}=0.0\right)$ of the annular section is significantly reduced, and the whole remaining curves in the compression-bending zone above the horizontal axis are also reduced, while those curves in the tension-bending zone below the horizontal axis are almost not changed. That means, the lack of core concrete area has a great influence on compression-bending bearing capacity of the cross section and has little effect on the tension-bending bearing capacity.

\section{EXAMPLES}

In order to demonstrate the application of the nomogram above, a circular section subjected to seven load cases is selected, and the cross section parameters for all load cases are: $r=250 \mathrm{~mm}, a_{s}=37,50 \mathrm{~mm}, \mathrm{C} 40, f_{c}=19,1$;
HRB400, $f_{y}=360$. For comparison, the calculations were carried out according to the method of this paper and in the reference [1] respectively. The transcendental equations given in reference [1] for determining the reinforcement area of circular cross section are:

$$
\left\{\begin{array}{c}
N=\alpha f_{c} A\left(1-\frac{\sin 2 \pi \alpha}{2 \pi \alpha}\right)+\left(\alpha-\alpha_{t}\right) f_{y} A_{s} \\
M=\frac{2}{3} f_{c} A r \frac{\sin ^{3} \pi \alpha}{\pi}+f_{y} A_{s} r_{s} \frac{\sin \pi \alpha+\sin \pi \alpha_{t}}{\pi} \\
\alpha_{t}=1.25-2 \alpha
\end{array}\right.
$$

where $A$ and $A_{s}$ are cross section area and total area of reinforcement respectively. The angle $\alpha$ in Fig. 11 is expressed in radians.

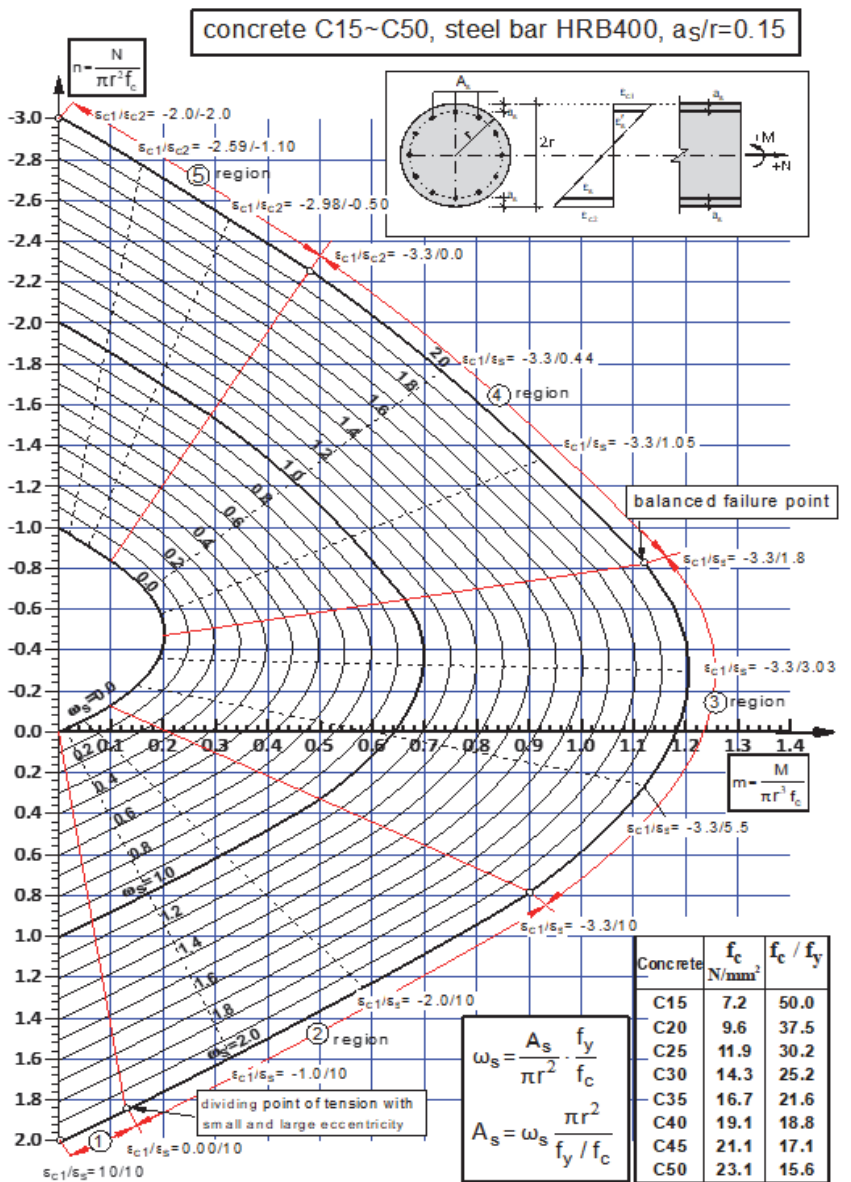

(a)

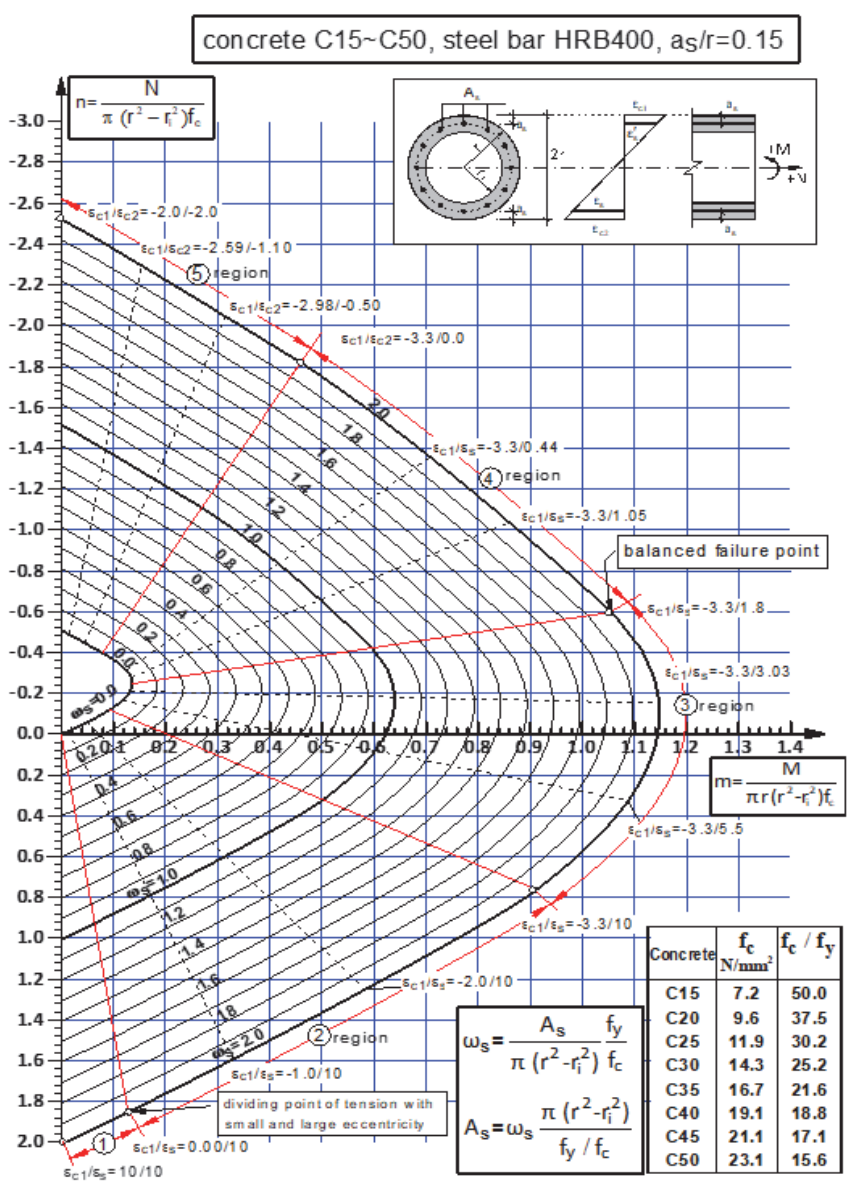

(b)

Figure 10 Nomogram of calculating reinforcement of circular and annular section

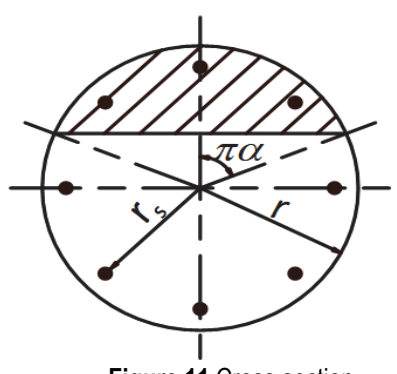

Figure 11 Cross section

The results calculated by both methods are shown in Tab. 3.

Comparing the calculation results of the two methods gives the following:

(1) 7 load cases in Tab. 3 can be easily calculated by using the design chart in Fig. 8. The calculation is very simple. Besides the determined reinforcement areas, the additional information such as strain distribution and strain region being able to be roughly determined for each load case can be also obtained, 
(2) Only 3 load cases in Tab. 3 could be solved by applying transcendental Eq. (23), which shows a good agreement with those of method 1, For remaining 4 load cases there is no solution obtained, because Eq. (23) is derived based on the fact that the neutral axis is located within the section.

\begin{tabular}{|c|c|c|c|c|c|c|c|c|c|c|}
\hline Example & Load cases & $\begin{array}{l}N / \\
\mathrm{kN}\end{array}$ & $\begin{array}{c}M / \\
\mathrm{kNm}\end{array}$ & $n=\frac{N}{\pi r^{2} f_{c}}$ & $m=\frac{M}{\pi r^{3} f_{c}}$ & $\varepsilon_{c 1} / \varepsilon_{S}$ & $\omega_{S}$ & $\begin{array}{c}\text { Method } 1 \\
A_{s}=\omega_{s} \frac{\pi r^{2}}{f_{y} / f_{c}} \\
/ \mathrm{cm}^{2}\end{array}$ & $\begin{array}{c}2 \\
\text { Method } 2 \\
A_{S} / \mathrm{cm}^{2}\end{array}$ & (2)/(1) \\
\hline 1 & uniform compression & -8250 & 0,0 & $-2,20$ & 0,00 & $-2,00 /-2,00$ & 1,20 & 12501 & - & - \\
\hline 2 & $\begin{array}{l}\text { compression with a } \\
\text { small eccentricity }\end{array}$ & -6750 & 187,5 & $-1,80$ & 0,20 & $-2,98 /-0,50$ & 1,12 & 11668 & - & - \\
\hline 3 & $\begin{array}{l}\text { compression with a } \\
\text { large eccentricity }\end{array}$ & -1500 & 843,8 & $-0,40$ & 0,90 & $-3,30 /+2,78$ & 1,40 & 14584 & 14112 & 0,967 \\
\hline 4 & pure bending & 0,0 & 562,5 & 0,00 & 0,60 & $-3,30 /+5,50$ & 0,93 & 9688 & 9478 & 0,978 \\
\hline 5 & $\begin{array}{c}\text { tension with a large } \\
\text { eccentricity }\end{array}$ & +1500 & 843,8 & 0,40 & 0,90 & $-3,30 /+7,50$ & 1,70 & 17710 & 17455 & 0,985 \\
\hline 6 & $\begin{array}{c}\text { tension with a small } \\
\text { eccentricity }\end{array}$ & +6000 & 93,75 & 1,60 & 0,10 & $+1,50 /+10,0$ & 1,71 & 17814 & - & - \\
\hline 7 & uniform tension & +3750 & 0,0 & 1,00 & 0,00 & $+10,0 /+10,0$ & 1,00 & 10417 & - & - \\
\hline
\end{tabular}

\section{CONCLUSIONS}

A method and the corresponding equations for calculating the reinforcement of circular and annular cross section is presented. Derivation of the equations is strictly based on the stress-strain relationship of concrete and reinforcement used in reference [1], which can be easily replaced by another stress-strain relationship of concrete and reinforcement given in Eurocode 2 or in ACI 318.

Dimensionless design charts - a useful tool for quick calculating reinforcement area of circle and annular cross section, are made. These design charts can be applied to all 7 load cases, such as uniform tension, tension with a small and large eccentricity, pure bending, compression with a large and small eccentricity and uniform compression, In addition, the design charts provide some other information, such as strain distribution, area of 5 strain regions, balanced failure point.

The core of the method is using strains to solve the stresses and then using stresses to solve the internal forces. After the all possible strain regions are determined in Fig. 2 , the strains become known variables, which can be given with different values and substituted into the relevant formulas. The stresses and internal forces can be finally obtained.

Fig. 10 is only applicable to ordinary concrete with grades C15 - C50, because their ultimate strains have the same value of $3.3 \%$. The ultimate strains of high-strength concrete (grades > C50) do not have the same constant value, so the design charts need to be recalculated and recreated, but the formulas derived in this article are still applicable.

\section{Acknowledgements}

This work was financially supported by the National Natural Science Foundation of China (51668027 and 51468026),

\section{REFERENCES}

[1] GB 50010. (2003).Code for design of concrete structures. Beijing, China Planning Press. (in Chinese).
[2] Shi, L. Q. \& Zhang, Y. X. (1991). Study Guide for concrete structure design guide. Beijing, Earthquake Press.

[3] Yuan, F. \& Yang, R. C. (1997). Theoretical formula and graphic solution for reinforcement calculation of columns with circular cross section. Journal of Nanjing Institute of architecture and Engineering, 1(1), 13-23.

[4] Wu, D. (2002). Concrete structure calculation manual.Beijing, China Architecture Building \& Press.

[5] Zhou, D. H. \& WANG, Q. F. (2010).A new method for the calculation of reinforcement of concrete: General dimensionless designing diagram. Engineering Mechanics, 27(1), 165-172. https://doi.org/10.3901/JME.2004.01.165

[6] Zhou, D. H. \& Cheng, H. M. (2007). A new kh0-method for calculation of singly reinforced concrete beam.Engineering Mechanics, 24(12), 106-112.

[7] Zhou, D. H., Cheng, H. M., etc. (2008). A new kh0-method for calculation of doubly reinforced concrete beam. Engineering Mechanics, 25(2), 188-192.

[8] EN1992 Eurocode 2. (2004). Design of concrete structures. Berlin,Beuth Verlag $\mathrm{GmbH}$.

[9] DIN 1045-1. (2008). Tragwerke aus Beton: Stahlbeton und Spannbeton. Berlin, Beuth Verlag GmbH.

[10] BS 8110-1. (1997). Structural use of concrete, Part 1: Code of practice for design and construction. London, UKBSI.

[11] Wieland, R. (2000). Vorlesungsscript für Massivbau und Baukonstruktion. Universität Kaiserslautern,Germany.

[12] Klaus, J. S. (2002). Bautabellen für Ingenieure. 15. Aufl., Düsseldorf, Werner Verlag.

[13] Tompert, K. (1971). Bemessung von Kreisquerschnitten und Kreisringquerschnitten nach dem Traglastverfahren. Bauingenieur, 46(3), 90-97.

[14] Gu, D. S., Wu, G., \& Wu, Z. S. (2012). Calculation of the ultimate flexural capacity of normal section of reinforced circular columns. Engineering Mechanics, 29(8), 227-235.

[15] Wu, M. J. \& Chen, S. Y. (2010). Study on simplified method of reinforcement calculation of circular section flexural members of reinforced concrete. Building Structure, 40(10), 102-104.

[16] Hsiao, K. J. (2012). Bending-Axis Effects on Load-Moment (P-M) Interaction Diagrams for CircularConcrete Columns Using a Limited Number of Longitudinal Reinforcing Bars. Electronic Journal of Structural Engineering, 12(1), 10-16.

[17] Quaranta, G.,Trentadue, F., \& Marano, G. C.(2017). Closedform approximation of the axial force-bending moment interaction diagram for hollow circular reinforced concrete cross-sections. Engineering Structures, 153(12), 516-524. https://doi.org/10.1016/j.engstruct.2017.10.042 
[18] Peng, K., Wang, Y.Q., Zou, Q., \& Liu, Z. (2019). Effect of crack angles on energy characteristics of sandstones under a complex stress path. Engineering Fracture Mechanics, 218(7), 1-11. https://doi.org/10.1016/j.engfracmech.2019.106577

[19] Du, P. W., Gan, D., Yang, Y. W., \& Tan, K. H. (2019) Simplified method for analysis of circular steel tubedreinforced-concrete columns under eccentric compression. Engineering Structures, 198(11). https://doi.org/10.1016/j.engstruct.2019.109524

\section{Contact information:}

Yao KAICHENG, PhD

Faculty of Civil Engineering and Mechanics, Kunming University of Science and Technology,

727 South Jingming Road, Chenggong District, Kunming, Yunnan 650500, China E-mail: ykc041430@qq.com

\section{Zhou DONGHUA, Prof., PhD}

(Corresponding author)

Faculty of Civil Engineering and Mechanics, Kunming University of Science and Technology,

727 South Jingming Road, Chenggong District, Kunming, Yunnan 650500, China E-mail: 806029639@qq.com

Shuang CHAO, PhD Postgraduates

Faculty of Civil Engineering and Mechanics, Kunming University of Science and Technology,

727 South Jingming Road, Chenggong District, Kunming, Yunnan 650500, China E-mail:1403094948@qq.com

\section{Lan SHUWEI, PhD Postgraduates}

Faculty of Civil Engineering and Mechanics, Kunming University of Science and Technology,

727 South Jingming Road, Chenggong District, Kunming, Yunnan 650500, China E-mail: 420113261@qq.com

Chen JIN, Assoc. Prof., MSc.

Faculty of Civil Engineering and Mechanics, Kunming University of Science and Technology,

727 South Jingming Road, Chenggong District, Kunming, Yunnan 650500, China E-mail: chen40333@yahoo.com.cn 